\title{
Research on Polymorphism Relationship of Th17/Treg Cell and Its Factors with STAT4 Gene in AITD Patients with Different lodine Nutritional Status
}

\author{
Feng Jing ${ }^{1}$, Mu Zhaoxin ${ }^{2}$, Hou Zhenjiang, ${ }^{2}$, Wang Cuicui ${ }^{2}$, Li Xinsheng', Chen Yunxia ${ }^{3}$, \\ Liu Jianfeng ${ }^{3}$, Ma Jinqun ${ }^{3}$, Liu Chunyan ${ }^{3}$ \\ ${ }^{1}$ Cangzhou Central Hospital, Cangzhou, China \\ ${ }^{2}$ Institute of Thyroid Diseases Affiliated to Cangzhou Medical College, Cangzhou Thyroid Disease Engineering Technology Research Center, \\ Cangzhou, China \\ ${ }^{3}$ Cangzhou People's Hospital Endocrinology, Cangzhou, China
}

Email address:

houzhenjiang@sina.com (Hou Zhenjiang)

${ }^{*}$ Corresponding author

\section{To cite this article:}

Feng Jing, Mu Zhaoxin, Hou Zhenjiang, Wang Cuicui, Li Xinsheng, Chen Yunxia, Liu Jianfeng, Ma Jinqun, Liu Chunyan. Research on Polymorphism Relationship of Th17/Treg Cell and Its Factors with STAT4 Gene in AITD Patients with Different Iodine Nutritional Status. American Journal of Clinical and Experimental Medicine. Vol. 9, No. 3, 2021, pp. 44-54. doi: 10.11648/j.ajcem.20210903.11

Received: April 2, 2021; Accepted: May 10, 2021; Published: May 24, 2021

\begin{abstract}
Background: Research has shown that STAT gene SNP is related to the pathogenesis of T1D, SLE, RA and AITD. Objective: To investigate the correlation of the SNP of Th17/Treg cell and its cytokines with STAT4 in the PBMC of AITD patients with different iodine nutritious status. Method: $100 \mathrm{GD}$ and HT patients who are selected from Cangzhou Central Hospital and Cangzhou People's Hospital from September 2019 to August 2020, respectively, 60 healthy cases, adopt flow cytometer and quantitative real-time PCR to test the Th17and Treg cell ratio and proportion in PBMC and the expression level of ROR- $\gamma$ t and 3 (Foxp3) mRNA, and use ELISA method to test IL-17 and TGF- $\beta$ level in the serum. Utilize SASP-PCR technology to test SNP locus (rs7574865) of STAT4 gene, and calculate the genotype and allele frequency. Result: There is some correlation between Th17/Treg cell \& its factors in PBMC in the AITD patients and STAT4 rs7574865; compared with healthy control group, the difference between STAT4 rs7574865 G/T \&T/T genotype and G\&T allele frequency is of statistic significance ( $\chi 2$ is 6.128 and 5.613 respectively, and the average value of $P$ is $<0.05$ ), and may increase the occurrence risk of AITD, but has no correlation with iodine nutritious status. Conclusion: AITD patients not only have Th17 and Treg cell imbalance and the corresponding factor changes but also correlate to STAT4 rs7574865 susceptibility, and the cellular immunity and gene SNP may participate in the disease occurrence process of AIDT together.
\end{abstract}

Keywords: Different Iodine Nutritional Status, Graves' Disease, Hashimoto Thyroiditis, Th17/Treg Cells and Factors, STAT4 Genes Polymorphism

\section{Introduction}

Autoimmune thyroid diseases (AITD) is a common organ specific Autoimmune diseases (AID), mainly including Graves' Disease (GD) and Hashimoto thyroiditis (HT), both of which have similar inheritance and immunity foundation, long time exposure to certain environmental factors may promote the population with specific gene background to suffer such a disease. The change of Universal Salt iodization (USI) in the past 10 years and next 10 years indicates that with the increase of iodine nutritious level, the occurrence rate of AITD also rises accordingly. GD is a common endocrine disease and has the highest occurrence rate among all the hyperthyroidism types, accounting for about $85 \%$ of the hyperthyroidism [1]. The occurrence rate of GD in western countries is about $0.5 \%-2 \%$, but for China, it is about 2\%-3.0\% [2]. The occurrence rate of $\mathrm{HT}$ in foreign countries is about $1 \%-2 \%$, accounting for $22.5 \%$ of thyroid diseases. However, in China, the occurrence rate of HT is about $0.3 \%-10 \%$, reaching as high as $22.5-40.7 / 100,000$ 
and including female and male of different ages. However, most patients are 30 to 50 years old, especially middle-aged women, accounting for about $90 \%$ [3]. The pathogenic factors for AITD are varied, and environment, inheritance and immunity imbalance, etc. may all cause the thyroid to have specific autoimmune response and induce the occurrence of HT [4], and make the patients have diffuse goiter or hyperthyroidism [5]. Environment, inheritance and autoimmune dysfunction, etc. may cause the thyroid to have specific autoimmune response, and make human body produce Thyroid peroxidase antibody (TPOAb), Thyroglobu-lin antibody ( $\mathrm{TgAb}$ ) and other autoantibodies, and make the patient have diffuse goiter, hyperthyroidism or hypermetabolic state, etc, and it attacks human of any age, but mainly females. Researches discover that many reasons cause the rise of DG occurrence, and iodine nutritious states is one of the influencing factors. The over take-in of iodine will increase the occurrence of GD and the positive rate of autoantibody of thyroid, and with the deterioration of the disease, the reoccurrence rate rises too [6]. Recent researches show that the main cytokines and specific transcrption factors secreted by Th17 and Treg cells are interleukin-17 (IL-17) \& transforming growth factor (TGF- $\beta$ ) andretinoic acid-related orphan receptors (ROR- $\gamma \mathrm{t}$ ) and Fork-head boxp3 (Foxp3) respectively, which play important roles in immunosuppression and have become the research direction for the occurrence, development and prognosis of diseases concerning immunity. In recent years, foreign countries have carried out wide researches for Th17/Treg cell and ROR- $\gamma$ t\& Foxp3 in RA and other AIDs. Many researches show that single nucleotide polymorphism (SNP) of signal transducers and activators of transcription (STAT) may correlate to the occurrence of type 1 diabetes (T1D) $[8,9]$, systemic lupus erythematosus (SLE) [10], reacting antibodies (RA) [11], AITD [12] and other AIDs, but the research results are varied. Joint test for Th17/Treg and its factors as well as SNP of STAT4 may be favorable for discussing the diagnosis, treatment, prevention and pathogenesis of AITD, and open a new channel for the research of immunogenetics of AITD.

\section{Materials and Methods}

\subsection{Research Object and Grouping}

Select 100 GD outpatient patients or inpatients who are preliminarily diagnosed but not treated yet of Endocrinology Department from Cangzhou Central Hospital and Cangzhou People's Hospital from September 2019 to August 2020 as the experiment group, including 26 male patients and 76 female patients, with the average age as $(45.4 \pm 15.1)$. All the patents conform to the GD diagnosis standards [13] of Guideline for the Diagnosis and Treatment of Thyroid Diseases in China formulated by Chinese Society of Endocrinology in 2008 and haven't got relevant treatment of Graves. As per the test result of thyroid hormone and corresponding clinic symptoms or signs, the experiment group is divided into GD-A group (clinical hyperthyroidism: have the symptom or sign of hyperthyroidism, and FT3 $>7.1 \mathrm{pmol} / \mathrm{L}, \quad \mathrm{FT} 4>22 \mathrm{pmol} / \mathrm{L}$,
$\mathrm{TSH}<0.27 \mathrm{mU} / \mathrm{L}$ ) and GD-B group (subclinical hyperthyroi -dism): without hyperthyroidism symptoms or signs, with normal FT3 and FT4, TSH<0.27mU/L). Exclusion standard: (1) Have other autoimmune system diseases, (2) Acute or chronic infection, malignant tumor, pregnancy or other allergy diseases, (3) History of diabetes, dysfunction of liver, blood system disease, (4) Patient who have had hyperglucocorticoid therapy in recent 30 days. $100 \mathrm{HT}$ patients are the experiment group, including 26 males and 74 females, with the average age as (47.4 \pm 16.8$)$. As per the examination result of thyroid function, HT patients are divided into group with normal thyroid function (HT-A group, 31 cases), subclinical hypothyroidism group (HT-B group, 46 cases) and clinical hypothyroidism group (HT-C group, 23 cases). All the patients conform to the HT diagnosis standards [14] of Guideline for the Diagnosis and Treatment of Thyroid Diseases in China formulated by Chinese Society of Endocrinology in 2008 and haven't got relevant treatment. HT inclusion standards: (1) $\mathrm{TSH}$ level is abnormal, anti-TPOAb and / or TGAb exceeds the upper limit of normal scope, (2) the thyroid texture is hard, with or without thyromegaly, (3) Have validation through aspiration cytologic or pathology examination after surgery. HT exclusion standards: (1) Recently, the patient has had systematic lupus erythematosis, rheumatoid arthritis, asthma, pernicious anemia, multiple sclerosis, systemic sclerosis, etc. (2) Recently ( $\leq 6$ months), the patient has had virus or bacteria infection history, (3) Recently ( $\leq 6$ months), the patients have had pregnancy or delivery history, (4) The patients have had malignant tumor history. Select 60 healthy cases with the gender and age matching in the same period as the control group (NC group), including 16 males and 44 females, with the average age as $(45.8 \pm 17.6)$, and the gender and age difference between groups has no statistic significance. The evaluation and analysis of iodine nutritious status adopts the standard issued by the WHO, UNICEF and ICCIDD in 2007. In case Median urinary iodine (MUI) is $<100 \mu \mathrm{g} / \mathrm{L}$, it is iodine lack, in case the MUI is $100-199 \mu \mathrm{g} / \mathrm{L}$, it is appropriate iodine amount, in case the MUI is $200-299 \mu \mathrm{g} / \mathrm{L}$, it is iodine overrunning, and in case MUI is $\geq 300 \mu \mathrm{g} / \mathrm{L}$, it is iodine excess [15]. This research is approved by the Ethics Committee and the research objects have signed informed consent form.

\subsection{Research Method and Items}

\subsubsection{Specimen Collection and Treatment}

(i) Specimen collection: all the research objects have a fast of more than 12 hours, keep 10 to $20 \mathrm{ml}$ of fasting midstream urine and place it in clean polyethylene pipe, and take $10 \mathrm{ml}$ of fasting heparin anticoagulation of venous blood from $8 \mathrm{am}$ to $10 \mathrm{am}$ and keep it in a refrigerator of $4^{\circ} \mathrm{C}$.(ii) PBMC separation: Use density gradient centrifugation to separate $\mathrm{PBMC}$, adjust the concentration density of the PBMC of each specimen as $1 \times 10^{6} / \mathrm{L}$, and suspend it in the culture solution of fetal calf serum of RPMI640, place the PBMC suspension in a 24-hole culture plate, add $25 \mu \mathrm{g} / \mathrm{L}$ of phorbol ester (American Alexis Company) and $1 \mathrm{mg} / \mathrm{L}$ of enomycin (American Alexis company) into each hole, and culture for $4 \mathrm{~h}$ in a $37^{\circ} \mathrm{C}$ incubator, and collect the cells for future use.(iii) RNA 
extraction and cNDA synthesis: Adopt Trizol method to extract the total RNA of PMBCs of the GD and HT patients and the control group, adopt denatured formaldehyde electrophoresis and absorbance value (A260/A280) to identify RNA quality. Take RNA with the ratio of A260/A280 above 1.9 and with the cataphoresis showing at least two zones to have the following experiment and adjust the concentration of each specimen RNA into $0.5 \mu \mathrm{g} /$ Lfor later use.

\subsubsection{Item Test}

(i) Urine iodine test: adopt the reaction principle of catalyzing arsenic and cerium with iodine, and test the urine iodine content by means of Arsenic cerium catalytic spectrophotometry (WS/T107-2006), the reagent kit is produced by ConsonBiochemicals and the instrument used is Hitachi 7600-110 automatic biochemical analyzer. (ii) Test for the ratio of Th17 and Treg cells: Use human Th17 cell testing kit (FITC/CD3, PE/CD4, Alexa Fluor647/IL-17, American Biolegend Company) to mark the TH17 cell, use human Treg cell testing kit (FOXP3 Alexa Fluor 488/CD4 PE-Cy5/CD25PE) to mark Treg cells, and use FACSCalibur flow cytometer (American BD company) for the following test: add the antibody marked with corresponding fluorescence dye into flow tube with $100 \mu \mathrm{L}$ of blood specimen as per the instruction manual, culture for $15 \mathrm{~min}$ in darkness under room temperature, add red blood cell lysis buffer, mix evenly and culture 10min in darkness, and add PBS solution and mix evenly, then run it on the instrument for testing, the testing result will be expressed in percentage and fluorescence intensity of positive cells and the analysis data will be obtained through CellQuest software. (iii) Adopt ELISA method to test serum IL-17 and TGF- $\beta$ level. (iv) PCR proliferation and genotyping: Adopt Trizol method to extract total RNA of the specimen, and synthesize cDNA as per reverse transcription procedure, extract DNA, apply PCR-SASP technology for rs 7574865 locus proliferation of STAT4 gene, with the forward primer as 5'-AGTATGAAAAGTTG-GTGAC-3' and reverse primer as 5'-AATCCCCTGAAATTCCACTG-3', with the primer synthesized by Shanghai Generay Biotech Co., Ltd., take $0.2 \mu \mathrm{g}$ DNA as the template, and have PCR proliferation in a reaction volume of $30 \mu \mathrm{L}$, with the reaction conditions as the following: Denature for $5 \mathrm{~min}$ under the temperature of $95^{\circ} \mathrm{C}$ and denature for 40 sunder the temperature of $95^{\circ} \mathrm{C}$, renature for $40 \mathrm{~s}$ under the temperature of $60^{\circ} \mathrm{C}$, extend for $40 \mathrm{~s}$ under the temperature of $72^{\circ} \mathrm{C}$, and extend for $7 \mathrm{~min}$ under the temperature of $72^{\circ} \mathrm{C}$ and then store, after 40 circulations, use agarose gel of $2 \%$ to have electrophoresis for the proliferation products, and identify the gene after dyeing, the PCR proliferation products will be completed and purified by primer synthesis company for the genotyping of SNP locus rs 7574865 of STAT4.

\subsubsection{Statistics Method}

Apply SPSS20.0 software for statistics analysis, the measurement data will be expressed with average \pm standard deviation $(\bar{x} \pm \mathrm{s})$, the comparison of the two sample average will adopt $\mathrm{T}$ inspection, the correlation analysis of two variables adopt Pearson linear correlation analysis, so as to compare the Th17/Treg ratio and activity of two groups of patients, as well as the expression level of Foxp3 and ROR- $\gamma \mathrm{t}$ mRNA. Have Hardy-Weinberg equilibrium inspection for each group genotype and allele distribution frequency, so as to confirm whether it conforms to the equilibrium law, and calculate the genotype and allele distribution frequency, and use odds ratio $[\mathrm{OR}=(\mathrm{a} / \mathrm{b}) /(\mathrm{c} / \mathrm{d})]$ to express relative danger degree, and compare through $\chi 2$ inspection, in case of $\mathrm{P}<0.05$, the difference is of statistic significance.

\section{Results}

\subsection{Comparison of Th17 and Treg Cells \&Its Cytokines with Transcription Factors Between the AITD Patients and the Control Group}

This Research result discovers that theTh17 cell ratio, Th17/Treg ratio, IL-17, TGF- $\beta$ and ROR- $\gamma$ t of AITD patients are higher than the NC group $(\mathrm{P}<0.05-<0.01)$, while Treg cell ratio and Foxp3 level is significantly lower than the control group $(\mathrm{P}<0.05)$. The Th17 cell ratio, Th17/Treg ratio, IL-17 and ROR- $\gamma$ t of the GD patients are all higher than the NC group $(\mathrm{P}<0.05-<0.01)$, while the ratio of Treg cell is lower than the control group $(\mathrm{P}<0.05)$, Foxp3 and TGF- $\beta$ are also lower than the control group, but the difference has no statistic significance. The GD patients' Th17 cell ratio, Th17/Treg ratio, IL-17, TGF- $\beta$ and ROR- $\gamma$ t are all higher than the NC group $(\mathrm{P}<0.05-<0.01)$, while Treg cell ratio and Foxp3 level are obviously lower than the control group $(\mathrm{P}<0.05)$. Th17 and Treg cell ratio, Th17/Treg ratio, and IL-17 of the GD patients are all higher than HT group, while TGF- $\beta$ and Foxp3 level are both lower than the HT group. The change to the Th17 and Treg cells \& their cytokines and transcription factors of GD patients are more significant than the HT patients, as shown in Table 1.

Table 1. Comparison of Th17 and Treg cells \& their cytokines and transcription factors beween AITD patients and the control group.

\begin{tabular}{|c|c|c|c|c|c|c|c|c|}
\hline Group & $\mathbf{N}$ & Th17cells\% & Tregcells\% & Th17/Treg & IL-17 (pg/ml) & TGF- $\beta(p g / m l)$ & ROR- $\gamma \mathrm{t}$ & Foxp3 \\
\hline AITD & 200 & $2.85 \pm 0.52^{\wedge}$ & $2.79 \pm 0.72 *$ & $1.36 \pm 0.47^{\Delta}$ & $35.7 \pm 6.51^{\wedge}$ & $21.04 \pm 3.29 *$ & $6.71 \pm 1.46^{*}$ & $1.31 \pm 0.23^{*}$ \\
\hline GD & 100 & $3.54 \pm 0.61^{\wedge}$ & $3.19 \pm 1.1^{*}$ & $1.76 \pm 0.51^{\wedge}$ & $36.3 \pm 5.65^{\wedge}$ & $14.09 \pm 4.7$ & $5.94 \pm 1.6^{*}$ & $1.76 \pm 0.24$ \\
\hline $\mathrm{NC}$ & 60 & $0.91 \pm 0.28$ & $6.73 \pm 0.97$ & $0.12 \pm 0.04$ & $13.17 \pm 2.84$ & $15.97 \pm 3.68$ & $1.49 \pm 0.38$ & $1.89 \pm 0.63$ \\
\hline
\end{tabular}

Note: Compared With Control Group, ${ }^{*} P<0.05,{ }^{\wedge} \mathrm{P}<0.01$. 


\subsection{Comparison of STAT4 rs7574865 Genotype and Allele Distribution Frequency Between the AITD Patients and the Control Group}

STAT4 rs7574865 genotype frequency distribution conforms to the Hardy-Weinberg law of genetic equilibrium, the STAT4 rs7574865 G/G, G/T, T/T genotype and G,T allele distribution frequency of the AITD patients and the control group are $39 \%, 44 \%, 17 \%$ and $61.3 \%, 38.7 \%$ as well as $45.0 \%$, $43.3 \%, 11.7 \%$ and $65.6 \%, 34.4 \%$ respectively, of which, G/T and $\mathrm{T} / \mathrm{T}$ genotype and $\mathrm{T}$ allele distribution frequency are higher than the healthy control group, while $\mathrm{G} / \mathrm{G}$ genotype and $\mathrm{G}$ allele distribution frequency are lower than the healthy control group, and the difference of each group is of statistic significance $(\mathrm{P}<0.05)$, as shown in Table 2 .

Table 2. Comparison of STAT4 rs 7574865 genotype and allele distribution frequency between the AITD patients and the control group.

\begin{tabular}{llll}
\hline & AITD & NC & $\boldsymbol{\chi}^{2}$ \\
\hline Genotype frequency & & & \\
G/G & $39(39 \%)$ & $27(45.0 \%)$ & 6.128 \\
G/T & $44(44 \%)$ & $26(43.3 \%)$ & \\
T/T & $17(17 \%)$ & $7(11.7 \%)$ & \\
Allele frequency & & $105(65.6 \%)$ & 5.036 \\
G & $98(61.3 \%)$ & $55(34.4 \%)$ & 0.027 \\
T & $62(38.7 \%)$ & & \\
\hline
\end{tabular}

\subsection{Comparison of STAT4 rs7574865 Genotype and Allele Distribution Frequency Between the GD Patients and the Control Group}

group is $38 \%, 49 \%, 13 \%$ and $61.9 \%, 38.1 \%$ as well as $45 \%$, $43.3 \%, 11.7 \%$ and $65.6 \%, 34.4 \%$ respectively, and the comparison difference between two groups is of statistics significance $(\mathrm{P}<0.05)$, as shown in Table 3 .

The STAT4 rs7574865 G/G, G/T, T/T genotype and G, T allele distribution frequency of the GD patients and the control

Table 3. Comparison of STAT4 rs7574865 genotype and allele distribution frequency between the GD patients and the control group.

\begin{tabular}{llll}
\hline & GD & NS & $\boldsymbol{\chi}^{2}$ \\
\hline Genotype frequency & & & \\
G/G & $38(38 \%)$ & $27(45.0 \%)$ & 5.184 \\
G/T & $49(49 \%)$ & $26(43.3 \%)$ & 0.039 \\
T/T & $13(13 \%)$ & $7(11.7 \%)$ & \\
Allele frequency & & & \\
G & $99(61.9 \%)$ & $105(65.6 \%)$ & 4.912 \\
T & $61(38.1 \%)$ & $55(34.4 \%)$ & 0.047 \\
\hline
\end{tabular}

\subsection{Comparison of Relative Danger Degree of STAT4 rs7574865 Genotype and Allele to GD}

Relative danger degree OR value of STAT4 $\mathrm{rs} 7574865 \mathrm{G} / \mathrm{G}$, $\mathrm{G} / \mathrm{T}, \mathrm{T} / \mathrm{T}$ genotype and $\mathrm{G}, \mathrm{T}$ allele is $0.749,1.257,1.132$ and $0.850,1.176$ respectively, and the relative danger degree of
G/T and T/T genotype to GD has statistic significance (all the $\mathrm{P}<0.05$ ), while the difference of relative danger degree of $\mathrm{G} / \mathrm{G}$ and $\mathrm{G}$ allele to GD has no statistic significance (all the $\mathrm{P}$ value $>0.05$ ), as shown in Table 4 .

Table 4. Comparison of relative danger degree of STAT4 rs7574865 genotype and allele to GD.

\begin{tabular}{|c|c|c|c|c|c|}
\hline & \multirow{2}{*}{ OR } & \multicolumn{2}{|c|}{$95 \%$ CI } & \multirow{2}{*}{$\chi^{2}$} & \multirow{2}{*}{$\mathbf{P}$} \\
\hline & & lower & upper & & \\
\hline \multicolumn{6}{|c|}{ Genotype frequency } \\
\hline $\mathrm{G} / \mathrm{G}$ & 0.748 & 0.259 & 2.436 & 11.182 & 0.052 \\
\hline $\mathrm{G} / \mathrm{T}$ & 1.257 & 0.472 & 1.638 & 10.326 & 0.046 \\
\hline $\mathrm{T} / \mathrm{T}$ & 1.132 & 0.318 & 2.012 & 12.509 & 0.038 \\
\hline \multicolumn{6}{|c|}{ Allele frequency } \\
\hline G & 0.850 & 0.289 & 1.178 & 2,513 & 0.417 \\
\hline $\mathrm{T}$ & 1.176 & 0.416 & 2.044 & 3.629 & 0.352 \\
\hline
\end{tabular}

\subsection{Comparison of STAT4 rs 7574865 Genotype and Allele Between HT Patients and the Control Group}

The STAT4 rs7574865 G/G, G/T, T/T genotype and G and T allele distribution frequency of the HT patients and the control group are $41 \%, 44 \%, 15 \%$ and $63.8 \%, 36.2 \%$ as well as $45 \%$, $43.3 \%, 11.7 \%$ and $65.6 \%, 34.4 \%$ respectively, and the comparison difference between two groups has statistic significance $(\mathrm{P}<0.05)$, as shown in Table 5. 
Table 5. Comparison of STAT-4 rs7574865 genotype and allele distribution frequency between the HT patients and the control group.

\begin{tabular}{lllll}
\hline & HT & NC & $\chi^{2}$ & P \\
\hline Genotype frequency & & & & \\
G/G & $41(41 \%)$ & $27(45.0 \%)$ & 5.813 & \\
G/T & $44(44 \%)$ & $26(43.3 \%)$ & & \\
T/T & $15(15 \%)$ & $7(11.7 \%)$ & 4.029 & \\
Allele frequency & & & & \\
G & $102(63.8 \%)$ & $105(65.6 \%)$ & & \\
T & $58(36.2 \%)$ & $55(34.4 \%)$ & \\
\hline
\end{tabular}

\subsection{Comparison of Relative Danger Degree of 6STAT4 rs7574865 Genotype and Allele to HT}

The relative danger degree OR value of STAT4 rs7574865 G/G, G/T, T/T genotype and G, T allele of the HT patients are $0.849,1.027,1.336$ and $0.921,1.085$ respectively. The relative danger degree difference of $\mathrm{G} / \mathrm{T}$ and $\mathrm{T} / \mathrm{T}$ genotype to HT has statistic significance (all the $\mathrm{P}<0.05$ ), while the relative danger degree of $\mathrm{G} / \mathrm{G}$ genotype and $\mathrm{G}$ and $\mathrm{T}$ allele to HT has no statistic significance (all $\mathrm{P}>0.05$ ), as shown in Table 6 .

Table 6. Comparison of relative danger degree of STAT4 rs 7574865 genotype and allele to HT.

\begin{tabular}{|c|c|c|c|c|c|}
\hline & \multirow{2}{*}{ OR } & \multicolumn{2}{|c|}{$95 \% \mathrm{CI}$} & \multirow{2}{*}{$\chi^{2}$} & \multirow{2}{*}{$\mathbf{P}$} \\
\hline & & lower & upper & & \\
\hline \multicolumn{6}{|c|}{ Genotype frequency } \\
\hline $\mathrm{G} / \mathrm{G}$ & 0.849 & 0.132 & 0.517 & 12.42 & 0.061 \\
\hline $\mathrm{G} / \mathrm{T}$ & 1.027 & 0.160 & 1.362 & 11.37 & 0.046 \\
\hline $\mathrm{T} / \mathrm{T}$ & 1.336 & 0.203 & 1.406 & 14.28 & 0.039 \\
\hline \multicolumn{6}{|c|}{ Allele frequency } \\
\hline G & 0.921 & 0.152 & 1.208 & 1.428 & 0.372 \\
\hline
\end{tabular}

\subsection{Comparison of STAT4 rs7574865 Genotype and Allele Distribution Frequency and Relative Danger Degree of AITD with Different Iodine Nutritious Status}

Compared with the control group, the difference of Th17/Treg ratio \& its cytokines (IL-17, TGF- $\beta$ ) and transcription factors (ROR- $\gamma \mathrm{t}$ and Foxp3) of the AITD patients with different iodine nutritious status, GD and HT patients has statistic significance $(\mathrm{P}<0.05)$, but the difference with the STAT4 rs7574865 genotype and allele distribution frequency and relative danger degree has no statistic significance $(\mathrm{P}>0.05)$.

\section{Discussion}

In recent years, researches validated that Th17 and Treg cell and its factors correlate to the occurrence of AITD and many other types of AIDs. Zhang, etc. [16] reported that the Th17 cell of GD patients rises significantly, while Treg cell drops significantly, which shows that Th17 and Treg cells play an important role in the occurrence of GD. Qin, etc. [17] discovered that GD patients' Th17 cell ratio, Th17/Treg ratio, and ROR- $\gamma \mathrm{t}$ and IL-17 gene expression level all rise significantly, while Treg and Breg ratio and Foxp3 gene expression level drops significantly, but IL-10 level shows no significant change. The rise of TH17 cell ratio and Treg cell drop in PBMC of HT hypothyroidism patients are more significantly than that of the people with normal thyroid function and subclinical hypothyroidism HT patients [18], which proves that thyroid dysfunction may affect the
Th17/Treg cell function. The Research result shows that AITD patients' Th17/Treg cells and their factors participate in the pathogenesis. Th17 cell ratio, serum IL-17 and ROR- $\gamma$ t in PBMC of GD patients rise significantly, while Treg cells and Foxp3 mRNA expression drop significantly, and TGF- $\beta$ also drops. The GD patients' Th17/Treg cells ratio is in significant positive correlation with TPOAb and TgAb titer, and its antibody titer has relatively big correlation with Th17/Treg, IL-17 and ROR-yt. Th17 cell ratio, Th17/Treg ratio, serum IL-17 and ROR- $\gamma \mathrm{t}$ in the PBMC of HT patients rise significantly, while Treg cell ratio, Th17/Treg ratio and Foxp3 mRNA expression level drop significantly, and show progressing changes at different stage of the disease, HT has Th17/Treg cell abnormality while the thyroid function is still normal, subclinical hypothyroidism patients have significant changes, compare hypothyroidism with subclinical hypothyroidism and normal thyroid function, the Treg cell in its PBMC is the lowest, while the TH17 cell ratio is the highest, with the deterioration of the disease, HT patients with hypothyroidism has the most significant changes, which shows that the HT patients' TH17 and Treg cell change has close relationship with the thyroid function, and TH17/Treg cell imbalance penetrates different stages of HT, which is consistent with the result of XueHaibo and Pyzik [18, 19]. It indicates that Th17/Treg cell imbalance and its factor change may play a role in the production of thyroid autoantibody and mediating $\mathrm{T}$ cell abnormal immune response, and participate in the autoimmunity damage of the thyroid tissue in return. It validates that different thyroid function itself may have some influence to the TH17 and Treg cell in PBMC, the imbalance 
of two may play an important role in HT occurrence [20]. It is estimated that the change of the HT patients' Th17 and Treg cell ratio at different stages may be caused by the change of the thyroid function, and it still deserves further discussion whether it has direct relationship with the immune response.

AITD is a typical group of organ specific AIDs mediated by $\mathrm{T}$ cells, and the global AITD occurrence rate gets close to 5\% [21]. AITD is mainly GD and HT whose features are hyperthyroidism and hypothyroidism respectively, and the lymphocytic infiltration of thyroid and generation of thyroid autoantibody, as well as family clustering. Among AITD, 20\% to $30 \%$ are the siblings of the patients, and the risk for siblings to have AITD is about $17 \%$, and the genetic risk is about $80 \%$. Gene data points out that shared genes and specific gene participate in the occurrence of GD and HT jointly [21], and the interaction of genetic susceptibility, immunity and environment factors is the key factor for the disease occurrence and development. In recent years, more and more researches disclose the role of genetic factors in AITD occurrence mechanism, SNP is one of the important aspects as well as the most common genetic variation of human, located at the coding region and promoter of the gene, affects gene functions in return, but its action mechanism is still not completely clear yet. SNP refers to the change of polymorphism of DNA sequence caused by two different basic groups on a certain ribotide position owing to conversion or reversal of a certain basic group of a single ribotide or the insertion or loss of one or several ribotide on genetic level. As a genetic unit, SNP widely exists in human DNA genomes and is one of the most common kinds in genetic variation, and accounts for more than $90 \%$ of the human gene DNA variation [22]. There is one SNP every 1000 basic groups, and it is estimated that its total number may reach 3 million or even more [23]. SNP exists not only in gene sequence but also in non-coding sequence beyond genes. The SNP in gene coding region, especially the SNP for coding immune response factor gene, may affect the transcription, expression of the genes or the structure of the coding protein [24], as the SNP locus genetic variation may increase the occurrence rate and susceptibility of certain diseases [25]. In recent years, the relationship between gene SNPs and diseases have attracted a lot of clinical attention, and become an emerging field of gene research. The gene susceptibility correlates to the occurrence of AID, and there is research [8-12] showing that STAT SNP participates in the occurrence of T1D and many other AIDs. Currently, the mechanism for SNPs to regulate STAT4 expression level is still not completely clear. Maybe rs7574865 affects the methylation level of STAT4 DNA and affects the expression level of STAT4 in return, and it is also possible to form different spliceosome STAT4 $\alpha$ and STAT4 $\beta$ or cut off the transcription factors or histone combination locus to complete such a mechanism [26].

Janus Kinase-Signal Transducers and Activators of Transcription (JAK-STAT) signal pathway is another important cytokine signal transduction pathway after Ras channel [27]. Currently, 4 types of JACK (including JAK1,
JAK2, JAK3and Tyk2) and 7 types of STAT (including STAT1-4, STAT5a, STAT5b and STAT6, etc.) have been cloned successfully [28]. STAT family all hashomeodomain of SH2and SH3, is able to combine with specific phosphorylated tyrosine peptide, have polymerization through phosphoryla -tion and convert into active activating transcription factor in the form of homodimer or heterodimer [29]. STAT4 is an important member of JAK/STAT signal pathway, and belongs to cell signal transducing molecules together with other 6 family members. Research discovered that STAT4 is a cytoplasmic factor located at NO. 2 long arm of chromosome (2q32.2-2q32.3) of human, with the coding sequence region composed of 2247 consecutive ribotides, with the length as about $120 \mathrm{~kb}$ and relative molecule weight as about $84 \mathrm{kD}$, coding 748 amino acids [30], includes 23 introns and 24 exons, the transcription factors coded by it transmits multiple intracellular signals [31]. STAT family is a protein which can combine with DNA, of which, STAT4 has different expression from other family members, has some tissue specificity, and is mainly distributed in lymphoid and bone marrow tissue [32]. STAT4 has close relationship with Th1 cell, and plays an important role in the differentiation and function of Th1 cells [33]. Coding transcription factors activate a series of inflammatory factors, and accelerate the differentiation of Th1 and Th17 cells, which causes the imbalance of Thl/Th2 cells in human body [34]. STAT4 is activated by the response induced by IL-12 [35], participates in mediating IL-12 and the regulation of downstream signal pathway of interferon- $\gamma$ and many other cytokines [36], and participates in the differentiation, proliferation and apoptosis of $\mathrm{T}$ cells by mediating the roles of IL-12, IL-23 and many other cytokines and growth factors signa 1 [26]. Activate IFN signal by affecting IL-12 and IL-23 pathway [26], and affect immunologic tolerance of normal human body. STAT4 not only regulates the balance between IL-12 and IL-23, but also affects the occurrence and development of inflammation by regulating the activity of Thl7and Thl cells [30]. Researches validated that many inflammations and AIDs are expressed as the imbalance of the above cytokines [37].

Many research results show that the polymorphism of STAT4 gene correlates to the occurrence of AIDs, STAT4 SNPs concerning AIDs include rs7574865, rs8179673, rs11889341, rs8179673, rs10181656, rs7582694, etc., which are all located in the $3^{\text {rd }}$ intron region of STAT4 and are not in the exon region [26]. Relatively many researches of Aids focus on the SNP locus rs7574865 of STAT4 gene. Some researches discover that STAT4 gene SNP correlates to the genetic susceptibility of T1D, AIH, SLE, RA, AITD and many other AIDs, but the research results are different and have disputes. STAT4 gene is one of the most interesting genes in the AIDs pathogenesis, including TID. Bi, etc [8] discovered thatrs 7574865 has relatively strong correlation with T1D gene $(\mathrm{P}<0.05)$, while rs3024866 has weak correlation with T1D gene. It is believed that SNP rs7574865 of STAT4 gene may play an important role in promoting the T1D susceptibility among Han nationality in Northeast China. Li, etc [37] discover that the haplotypes of rs $7582694 \mathrm{C}$ and rs7574865T 
allele can increase the occurrence rate of Type 1 AIH $(\mathrm{P}<0.001)$.

Yang, etc [38] had research for SNP of STAT4 gene for 2350 Chinese (SLE=910 cases, and healthy control cases $=1440$ cases) and 660 Thai people (SLE=278 cases, and control cases $=383$ cases) living in Hong Kong and discovered that STAT4 rs7574865 could increase the occurrence rate of SLE by 1.71 times, which indicates that STAT4 rs7574865 correlates to the occurrence of SLE. Hellquist, etc [39] had genotyping for 273 affected testees from 192 families from Finland and their health family relatives, and discovered that SNP rs7582694 and rs10181656 of STAT4 gene of Caucasian population correlate to SLE and can increase the occurrence risk of SLE. Hamad [40] etc. discovered that TT genotype and $\mathrm{T}$ allele frequency of RA patients are significantly higher than the control group, and the research result supports that SNP of STAT4 gene correlates to the genetic susceptibility of RA of Tunisian population. Namjou, etc. [10] collected 9923 SLE patients and control testes from different nationalities for STAT4 SNP research, and discovered that several SNPs correlate to the occurrence of SLE, SLE has strong genetic correlation in different nationalities, and STAT4 may be a key element in the SLE pathogenesis of multiple nationalities and play an important role in SLE occurrence. Du Jinge, etc. [30] had Meta analysis for 21902 SLE patients and 37780 control cases in 27 papers listed into research, and discovered that all the rs7574865 of all sub-groups of allele, homozygote type, heterozygote type, dominant inheritance and latent inheritance correlate to SLE. The result prompted that STAT4 rs7574865 locus SNP correlates to SLE susceptibility, and T allele may increase the occurrence rate of SLE. Wu Ximei, etc [41] discovered that SLE patients' G allele frequency of rs 7574865 locus of STAT4 gene is obviously lower than the normal control group, while $\mathrm{T}$ allele frequency is obviously higher than the normal control group, and the difference has statistic significance $(\mathrm{P}<0.05)$. SLE patients' GG homozygote type ratio is significantly lower than the healthy control group, while GT homozygote type ratio is obviously higher than the healthy control group, and the difference has statistic significance $(\mathrm{P}<0.05)$. The result shows that STAT4 gene rs7574865 correlates to the occurrence of SLE and its clinical features, and prompts that STAT4 SNP may participate in the occurrence and development process of SLE. The above research results are basically consistent both domestic and abroad and it is believed that STAT4 rs7574865 locus SNP correlates to SLE.

One control gene genotyping research [42] for 1620 cases of RA and 2635 control large samples in North America discovered that 4 SNPs, i.e. rs7574865, rs8179673, rs11889341 and rs 10181656 in the $3^{\text {rd }}$ intron region of STAT4 are in significant correlation with RA occurrence, and rs7574865 has the most significant correlation with the occurrence of RA. Hamad, etc [40] discovered that RA patients' TT genotype and T allele frequency are significantly higher than the healthy control group, and supports that SNP of STAT4 gene participates in the occurrence of RA in Tunisian population. $\mathrm{Gu}$, etc [43] had Meta analysis for
TT/GT+GG genotype, GT+TT/GG genotype, TT/GG genotype and T allele of 25300 RA patients and 26326 control groups listed into 17 case-control studies, and discovered that RA has correlation with STAT4 rs7574865 TT, GT+TT genotype and T allele, and the TT and GT+TT genotype and $\mathrm{T}$ allele of Europe, Asia, Africa, and Latin America are in significant correlation with RA, which may be independent from the existence of RF and anti CCP antibody. Ciccacci, etc [44] reported that STAT4 rs7574865 increases the RA susceptibility of Italian population and is in significant correlation with ACPA level. Durán-Avelar, etc [45] discovered that GT and TT genotype and T allele of STAT4 rs7574865 are in correlation with hazardous factors of RA, as well as with middle and high disease activities (DAS28 $\geq 3.2$ ), the HAQ value of patients carrying GT or TT genotype is lower than patients carrying GG genotype, which prompts that STAT4 rs7574865 correlates to RA activities, but doesn't support that the correlation of RA with anti CCP antibody level of Mexican population. E1, etc [11] reported that RA patients' STAT4 SNP GT+TT genotype and allele frequency are significantly higher than the control group, and correlate to RA. The RF and anti CCP positive rate of RA patients carrying $\mathrm{T}$ allele is significantly higher than RA patients carrying wild genotype, which proves that STAT4 rs7574865 is in correlation with RA of Egyptian population as well as with RF and anti CCP antibody positive. Ebrahimiyan, etc [46] had Meta analysis and evaluation for the correlation betweenSTAT4 rs7574865 and RA risk for 15732 cases of RA and 15641 healthy testes from 16 papers for 21 population studies, discovered that STAT4 rs7574865 is in positive correlation with RA occurrence risk, and disclosed the relationship between STAT4 rs7574865 and RA risk. Tarakji, etc [31] reported that the comparison difference of STAT4 rs7574865 genotype and allele frequency of ACPA positive \& ACPA negative patient with healthy control group has no statistic significance $(\mathrm{P}>0.05)$, and prompted that STAT4 rs7574865 genotype has no relationship with RA patients' susceptibility in Iran. Asiani, etc. [47] discovered that the detection rate ofrs7574865 SNP is lower than the control group, while the detection rate of $\mathrm{G}$ allele is relatively high, but the comparison difference with the control group has no statistic significance, and it is believed that rs7574865 has no correlation with the children's RA susceptibility in Iran. Most overseas research results believed that STAT4 rs7574865 has relationship with RA occurrence risk. However, there are also reports that STAT4 rs7574865 has no relationship with RA sussceptibility. Therefore, there may be susceptibility difference in different regions.

China also carried out correlation researches between START4 SNPs and RA. Xia Yan, etc [48] discovered that the difference between RA patients and healthy control group rs7574865 locus genotype frequency has statistic significance, TT genotype and T allele are both predisposing factors of RA, meanwhile dominant and latant models also display that rs 7574865 correlates to the susceptibility of RA occurrence, but rs2910164 locus has no relationship with RA susceptibility. Yang Meijuan, etc [49] reported that risk for RA patients 
carrying rs7574865 GT and TT to have RA increases significantly $(\mathrm{P}<0.05)$, which shows that $\mathrm{rs} 7574865$ correlates to the RA susceptibility of population in the northwest of Lanzhou. Wei Meng, etc [50] had genotyping for 23 SNPs locuses ofSTAT4 of 456 cases in soutwest Chengdu (288 RA patients and healthy control cases respectively), and discovered that the difference of genotype and allele frequency of rs11685878, rs12988825 and rs16833437 between 2 groups has statistic significance $(\mathrm{P}<0.05)$ and prompted that the genotype and allele frequency of several SNP locuses of STAT4 correlate to RA of Han nationality of China. Liang Aifeng, etc [51] reported that the comparison difference of GG, GT and TT3 genotype and G and T allele frequency of STAT4 rs7574865 locus between RA patients and the control group has no statistic significance $(\mathrm{P}>0.05)$, and prompted that STAT4 rs7574865 may have no relationship with the RA susceptibility of Guizhou population. $\mathrm{Xu}$ Zhenxin, etc [52] discovered that different allele frequency of rs3761847 and rs2228145 in Han nationality population in Xiamen is in significant correlation with RA susceplibility $(\mathrm{P}<0.05)$, while rs7574865, rs2234067and rs2230926 has no signicificant correlation with RA suscepibility $(\mathrm{P}>0.05)$. Most of the above research results show that STAT4 rs7574865 locus has relationship with the susceptibility of RA occurrence, but in some regions, there were also reports that different STAT4 SNP locuses have no relationship with the susceptibility of RA occurrence. Therefore, it is very important to research the relationship of STAT4 SNP with the RA susceptibility in different regions. Besides, STAT4 rs7574865 SNP can increase the RA and SLE occurrence risk for American, Japanese, Columbians and Mexicans.

In China and other countries, there are relatively many researches on the relationship of STAT4 rs7574865 SNP with SLE and RA, but few reseaches on AITD. In 2011, Park, etc [53] reported that STAT4 SNPs: rs11889341, rs7574865, rs8179673 and rs10181656 all can increase the AITD occurrence risk of South Korea population. Yan, etc [12] had genotyping forSTAT4 SNP: rs7574865, rs10181656 and rs7572482 of 1048 AITDs patients $(\mathrm{GD}=693, \mathrm{HT}=355)$ and 909 control cases and discovered that GD patients' rs 7574865 and rs10181656 genotype frequency has significant difference with the control group ( $\mathrm{P}=0.028$ and $\mathrm{P}=0.012)$, and $\mathrm{T}$ and $\mathrm{G}$ allele frequency is also significantly higher than the control group $(\mathrm{P}=0.020$ and $\mathrm{P}=0.031)$. The $\mathrm{GD}$ and HT patients' haplotype detection rate are significantly lower than the control group $(\mathrm{P}=0.015$ and 0.030$)$, while GD and HT patients haplotype GT is significantly higher than the control group $(\mathrm{P}=0.016$ and 0.048$)$. The result shows that $\mathrm{rs7574865}$ and rs10181656 SNP increases Chinese risk for having AITD. Zhao Jingya, etc [54] had genotype analysis for STAT4 rs7574865 for 61 GD patients and 50 healthy control cases, and discovered that the occurrence risk frequency of $\mathrm{G}$ and $\mathrm{T}$ allele is 1.452 times and 1.774 times of the control group, and prompted that STAT4 gene SNP has relationship with GD occurrence. Gao, etc [55] had Meta analysis for the website data of Embase, Pub Med, Web of Science and China National
Knowledge Infrastructure, had 1707 cases of AIDT and 3216 control cases of 5 independent cases listed in, and discovered that STAT4 rs7574865 is in significant correlation with AITD susceptibility [TT vs GG: $\mathrm{OR}=1.63,95 \% \mathrm{CI}=1.24-2.15, \mathrm{PZ}=$ 0.0005 , TT vs.(TG+GG) : $\mathrm{OR}=1.55,95 \% \mathrm{CI}=1.26-1.91$, $\mathrm{PZ}<0.0001]$. It indicates that, in Asian population, STAT4 rs7574865 is in significant corelation with the susceptibility of GD and HT occurrence, while not with African population. Hama, etc [40] had genotyping analysis for STAT4 rs7574865 SNP of 159 AITDs patients and 200 health control cases, and didn't discover that STAT4 rs7574865 SNP has significant correlation with AITD risk, and didn't support that STAT4 gene causes genetic susceptibility of AITDs to Tunisian population. This research shows that AITD patients' STAT4 rs $7574865 \mathrm{G} / \mathrm{T}$ and $\mathrm{T} / \mathrm{T}$ genotyping and $\mathrm{T}$ allele frequency are higher than the healthy control group, while G/G genotype and $\mathrm{G}$ allele frequency are lower than the healthy control group, and $\chi 2$ is 6.12 and 5.613 respectively, the average value of $P$ is $<0.05$, which prompts that STAT4 rs 7574865 has relationship with AITD occurrence. $\chi 2$ of G/T and T/T genotype and G and T allele frequency of GD and HT patients' STAT4 rs7574865 are 5.184, 4.912 and 5.813, 4.392 respectively, with the average value $<0.05$. The carried heterozygote GT and homozygote TT of STAT4 rs7574865 may be the dangerous factors for GD and HT occurrence, and the OR value are 1.257, 1.132 and $1.027,1.336$ respectively, which may increase the risk of GD and HT occurrence. However, the carried G/G genotype may be the protective factors for GD and HT occurrence, which may reduce the occurrence rate of GD and HT. Compared with the health control group, the drop of GD patients' GG genotype frequency $(\mathrm{OR}=0.749)$ is more significant than $\mathrm{HR}$ patients $(\mathrm{OR}=0.849)$, and the rise of $\mathrm{T}$ allele gene frequency $(\mathrm{OR}=1.176)$ is more significant than $\mathrm{HT}$ patients $(\mathrm{OR}=1.085)$. It can be concluded that STAT4 rs 7574865 correlates to the occurrence of GD and HT, and players a more important role in the occurrence of GD than HT. The result of this research is basically consistent with most of the above results, which indicates that STAT4 rs7574865 has correlation with the occurrence risk of AITD in Hebei which in the east of China, and is the susceptible gene for AITD occurrence, enriches the data of STAT4 gene SNP in AIDs Research, and may provide basis for prevention and control of AITD in the region. However, owing to the limit of insufficient specimens, the result deserves further validation.

AITD is a common clinical AID and its immunity and inheritance factors may play an important role in its occurrence. Most researches result shows that STAT4 rs 7574865 can increase the occurrence risk of AITD, but there are also different research results show that it may have relationship with inheritance background, habitant environment, and nationality differences and other factors, With the constant deepening of researches for human genetic genes, it discovers that the polymorphism change of multilple genes and multiple locuses have close relationship with the occurrence of AIDs. Therefore, it is an inevitable trend to carry out researches for SNP of STAT4 genes and discuss the mutual influence and interaction mechanism of Th17/Treg 
cells and its factors with SNP of STAT4 gene for the effective prevention and treatment of AITD and laying foundation of immunogenetics for the prevention and control of relevant diseases.

\section{Conclusion}

By carrying out correlation research of Th17/Treg cell and its factors with STAT4 gene SNP in AITD patients with different iodine nutritious status, it discovers that the Th17cell ratio, serum IL-17and ROR- $\gamma$ t in the PBMC of GD and HT patients with different iodine nutrious status rise significantly, while Treg cell ratio and Foxp3mRNA expression drop significantly, and the serum TGF- $\beta$ also drops. Th17/Treg cell ratio is in significant positive correlation with TPOAb and TgAb titer, and the antibody titer has relatively big correlation with Th17/Treg, IL-17 and ROR- $\gamma$ t. The comparison difference of the AITD patients' STAT4 rs7574865 GG, GT, TT genotype and G and T allele distribution frequency with the healthy control group has statistic significance $(\mathrm{P}<0.05)$, the one carrying heterozygote type GT and homozygote type TT has increased risk for having GD and HT (all the Ps $<0.05$ ), which prompts that rs 7574865 SNP correlates to the susceptibility of AITD in population in Hebei. AITD patients' Th17/Treg cells and factors correlate to STAT4 gene SNP, and may participate in the occurrence of the disease together, and are the immune and genetic factors affecting the occurrence and development of AITD. However, the difference of STAT4 rs7574865 genotype and allele distribution frequency between the AITD patients with different iodine conditions and the control group has no statistic significance, which indicates that iodine nutritious status has no correlation with STAT4 rs7574865 in the occurrence of AITD, but large sample farsightedness research needs to be further studied, so as to screen more AITD susceptible genes and elaborate the role of Th17/Treg cells and factors and STAT4 rs7574865 in the occurrence of AITD. It expects to provide basis for the early diagnosis, precise treatment, as well as the exploration and research for the mutual influence and interaction of cell immunity and gene genetics and open a new channel for scientific prevention and control from the perspective of cellunar immunity and molecular genetics.

\section{Acknowledgements}

Cangzhou Key R \& D Plan Guidance Project (Project Number: 1833020011).

Natural Science Foundation of Cangzhou Medical College (No. 18Z015).

\section{References}

[1] Chen X, Mei Y, He B, et al. General and specific genetic polymorphism of cytokines-related gene in AITD [J]. MediatorsInflamm, 2017, 2017 (1): 3916395.
[2] Xue L, Pan $\mathrm{C}, \mathrm{Gu} \mathrm{Z}$, et al. Genetic heterogeneity of susceptibility gene in different ethnic populations: refining association study of PTPN22 for Graves'disease in a Chinese Han population [J]. PLoS One. 2013, 8 (12): e84514.

[3] Xiang Guangda. Clinical Thyroidology [M]. People's Medical Publishing House, 2013: 200.

[4] Hu S, RaymanMP. Multiple Nutritional Factors and the Risk of Hashimoto's Thyroiditis [J]. Thyroid. 2017, 27 (5): 597-610.

[5] Li HX, Xiang N, Hu WK, et al. Relation between therapy options for Graves' disease and the course of Graves' ophthalmopathy: a systematic review and meta-analysis [J]. J Endocrinol Invest, 2016, 39 (11): 1225-1233.

[6] Su JP, Su SO, Zhang B, et al. The effects of different a mounts of iodine in take on the immunestatus of patients with Graves disease [J]. Clin Med China, 2012, 28 (1): 44-46.

[7] Hou ZJ, Mu ZX, Wang CC. Research Progress of Th17/Treg Cells and Their Transcription Factors in Autoimmune Diseases $[\mathrm{J}]$. American Journal of Clinical and Experimental Medicine 2019, 7 (4): 83-92.

[8] Bi C, Li B, Cheng Z, et al. Association study of STAT4 polymorphisms and type 1 diabetes in Northeastern Chinese Han population [J]. Tissue Antigens. 2013, 81 (3): 137-140.

[9] Fichna Marta, Żurawek Magdalena, Bogusz-Górna Klaudia, et al. STAT4 sequence variant and elevated gene expression are associated with type 1 diabetes in Polish children. Central-European J Immunology, 2020, 45 (1): 22-28.

[10] Namjou B, Sestak AL, Armstrong DL, et al. High-density genotyping of STAT4 reveals multiple haplotypic associations with systemic lupus erythematosus in different racial groups [J]. Arthritis Rheum, 2014, 60 (4): 1085-1095.

[11] El Lebedy D, Raslan H, Ibrahim A, et al. Association of STAT4 rs7574865 and PTPN22 rs2476601polymorphisms with rheumatoid arthritis and non-systemically reacting antibodies in Egyptian patients [J]. Clin Rheum, 2017, 36 (9): 1981-1987.

[12] Yan N, Meng S, Zhou J, et al. Association between STAT4 gene polymorphisms and autoimmune thyroid diseases in a Chinese population [J]. Int J Mol Sci, 2014, 15 (7): 12280-12293.

[13] Chinese Medical Association Endocrine Credits "China thyroid disease treatment guidelines" Writing Group. Chinese Guidelines for the Diagnosis and Treatment of thyroid diseases-Hyperthyroidism [J]. Chin J Intern Med, 2007, 46 (10): 876-882.

[14] Chinese Medical Association Endocrine Credits "China thyroid disease treatment guidelines" Writing Group. China thyroid disease treatment guidelines-Thyroiditis [J]. Chin J inter Med, 2008, 47 (9): 784-788.

[15] Chinese Medical Association Endocrine Credits "China thyroid disease treatment guidelines" Writing Group. China thyroid disease treatment guidelines-iodine deficiency disorders [J]. Chin J Intern Med, 2008, 47 (8): 689-690.

[16] Zhang DH, Qiu XG, Li JH, et al. MiR-23a-3p-regulated abnormal acetylation of FoxP3 induces regulatory $\mathrm{T}$ cell function defect in Graves' disease [J]. Biol Chem. 2019, 24, 400 (5): 639-650. 
[17] Qin J, Zhou J, Fan CL, et al. Increased Circulating Th17 but Decreased $\mathrm{CD}^{+}{ }^{+}$Foxp $33^{+}$Treg and $\mathrm{CD} 19^{+} \mathrm{CD} 1 \mathrm{~d}$ hi $\mathrm{CD}^{5+}$ Breg Subsets in New-Onset Graves' Disease [J]. Biomed Res Int. 2017, 2017: 8431838.

[18] Xue HB, Ma L, Li YB, et al. Correlation between Treg / Th17 cell and autoimmunity of Hashimoto's thyroiditis $[\mathrm{J}]$. China Journal of Modern Medicine, 2012, 22 (23): 67-71.

[19] Pyzik A, Grywalska E, Matyjaszek-Matuszek B, et al. Immune disorders in Hashimoto's thyroiditis: what do we know so far? [J]. J Immunol Res. 2015, 2015: 979167.

[20] González-AmaroR, Marazuela M. T regulatory (Treg) and T helper17 (Th17) lymphocytes in thyroid autoimmunity [J]. Endocrine. 2016, 52 (1): 30-38.

[21] Antonelli A, Ferrari SM, Corrado A, et al. Autoimmune thyroid disorders [J]. Autoimmun Rev, 2015, 14 (2): 174-180.

[22] XuJ. Correlation between polymorphisms of STAT4 rs7574865 and hepatitis B virus in fection related hepatocellular carcinoma [J]. Chine J Liver Disea (Electronic Version), 2019, $11(2): 10-14$.

[23] Lopes MS, Silva FF, Harlizius B, et al. Improved estimation of inbreeding and kinship in pigs using optimized SNP panels [J]. BMC Genet, 2013, 14: 92.

[24] Bogunia-Kubik K, Łacina P. From genetic single can didategene studies to complex genomics of GvHD [J]. Br J Haematol, 2017, 178 (5): 661-675.

[25] Kim LH, Cheong HS, Namgoong S, et al. Replication of genome wide association studies on hepatocellular carcinoma susceptibility loci of STAT4 and HLA-DQ in a Korean population [J]. Infect Genet Evol, 2015, 33: 72-76.

[26] Zhang W, Yang LH. Research progress on the association between STAT4 polymorphism and autoimmune diseases [J]. Chin J Immun, 2014, 30 (1): 141-144.

[27] Lin L, Li XJ, Wei XL. The mechanism of STAT4 and its related research in disease [J]. J Cell Mol Immunol, 2007, 23 (8): 784-786

[28] Liu X, Sa YL. Research Status and Progress of STAT4 Gene in Liver Diseases [J]. China J Med Guide, 2018, 20 (7): 401-405.

Ignacio A, Breda CNS, Camara NOS. Innate lymphoid cells in tissue homeostasis and diseases [J]. World J Hepatol. 2017, 9 (23): 979-989.

[29] Du JG, Chen H, Yang XR, et al. Meta-analysis of the association between the STAT4 rs7574865 polymorphism and systemic lupus erythematosus [J]. J Shan Dong Unive (HEALTH SCIENCES), 2017, 55 (5): 95-102.

[30] Tarakji I, Habbal W, Monem F. Association Between STAT4 rs7574865 Polymorphism and Rheumatoid Arthritis: Debate Unresolved [J]. Open Rheumatol J. 2018, 12: 172-178.

[31] Shi ZY, Zhang Q, Chen HX, et al. STAT4 polymorphisms are associated with neuromyelitisoptica spectrum disorders [J]. Neuromolecular Med, 2017, 19 (4): 493-500.

[32] Varikuti S, Oghumu S, Natarajan G, et al. STAT4 is required for the generation of Th1 and Th2, but not Th17 immune responses during monophosphoryl lipid A adjuvant activity [J]. Int Immunol, 2018, 30 (8): 385.

[33] Horwitz DA, Bickerton S, Koss M, et al. Suppression of
Murine Lupus by $\mathrm{CD} 4^{+}$and $\mathrm{CD} 8^{+}$Treg Cells Induced by $\mathrm{T}$ Cell-Targeted Nanoparticles Loaded With Interleukin-2 and Transforming Growth Factor $\beta[\mathrm{J}]$. Arthritis \& Rheumatol. 2019, 71 (4): 632-640.

[34] Enerbäck C, Sandin C, Lambert S, et al. The psoriasis-protective TYK2 I684S variant impairs IL-12 stimulated pSTAT4 response in skin-homing $\mathrm{CD}^{+}$and CD8 ${ }^{+}$memory T-cells [J]. Sci Rep. 2018, 8 (1): 7043.

[35] Xu LL, Ding SL, Zhang WW, et al. STAT4 deficiency exacerbates foam cell formation and atherosclerosis via MiR-9-de-pendent pathway in ApoE ${ }^{-/}$mice [J]. Chin J Arterioscler, 2020, 28 (5): 410-411.

[36] Li Xiaofeng, Chen Huiqin, Cai Yun, et al. Association of STAT4 and PTPN22 polymorphisms and their interactions with type-1 autoimmune hepatitis susceptibility in Chinese Han children. [J] Oncotarget, 2017, 8 (37): 60933-60940.

[37] Yang W, Ng P, Zhao M, et al. Population differences in SLE susceptibility genes: STAT4 and BLK, but not PXK, are associated with systemic lupus erythematosus in Hong Kong Chinese [J]. Genes Immun, 2009, 10 (3): 219-226.

[38] Hellquist A, Sandling JK, Zucchelli M, et al. Variation in STAT4 is associated with systemic lupus erythematosus in a Finnish family cohort [J]. Ann Rheum Dis, 2010, 69 (5): 883-886.

[39] Hamad MB, Cornelis F, Mbarek H, et al. Signal transducer and activator of transcription and the risk of rheumatoid arthritis and thyroid autoimmune disorders [J]. Clin Exp Rheumatol, 2011, 29 (2): 269-274.

[40] Wu XM, Hu QX, Li Bo, et al. Association of STAT4 rs7574865 polymorphism with systemic lupus erythematosus [J]. J Hainan Med, 2018, 29 (21): 3013-3014.

[41] Remmers EF, Plenge RM, Lee A, et al. STAT4 and the risk of rheumatoid arthritis and systemic lupus erythematosus [J]. N Engl J Med, 2007, 357 (10): 977-986.

[42] Gu E, Lu J, Xing D, et al. Rs7574865 polymorphism in signaltransducers and activators of transcription 4 gene and rheumatoid arthritis: an updated meta-analysis of 28 case-control comparisons [J]. Int J Rheum Dis, 2015, 18 (1): $3-16$.

[43] Ciccacci C, Conigliaro P, Perricone C, et al. Polymorphisms in STAT-4, IL-10, PSORS1C1, PTPN2 and MIR146A genes are associated differently with prognostic factors in Italian patients affected by rheumatoid arthritis [J]. Clin Exper Immunol, 2016, $186(2): 157-163$.

[44] Durán-Avelar MJ, Vibanco-Pérez N, Hernández-Pacheco RR, et al. STAT4 $\mathrm{rs} 7574865 \mathrm{G} / \mathrm{T}$ polymorphism isassociated with rheumatoid arthritis and disease activity, but not with anti-CCP antibody levels in a Mexicanpopulation [J]. Clin Rheumatol, 2016, 35 (12): 2909-2914.

[45] Ebrahimiyan H, Mostafaei S, Aslani S, et al. Studying the association between STAT4 gene polymorphism and susceptibility to rheumatoid arthritis disease: An updated meta-analysis [J]. Iran J Immunol, 2019, 16 (1): 71-83.

[46] Asiani S, Mahmoudi M, Salmaninejad A, et al. Lack of association between STAT4 single nucleotide polymorphisms and iranian juvenile rheumatoid arthriis patients [J]. Fetal Pediatr Pathol, 2017, 36 (3): 177-183. 
[47] Xia Y, Song JL, Feng J, et al. STAT4 rs7574865 polymorphism is associated with the susceptibility of rheumatoid arthritis inWuling mountain area [J]. Chin J Cell Mol Immunol, 2018, 34 (9): 829-833.

[48] Yang MJ, Zhang D, Zhang M, et al. Correlation between the genetic polymorphisms of STAT4 andPSORS1C1 and the susceptibility to rheumatic diseases in Han population of Lanzhou [J]. J Immun, 2018, 34 (7): 618-624.

[49] Wei M, Xie QY, Wang T, et al. Association of variants in signal transducer and activator of transcription 4 in Chinese Han population with rheumatoid arthritis [J]. Chin J Rheumatol, 2016, (2): 83-87.

[50] Liang AF, Fei Y, Cheng P, et al. Study on the Correlation of STAT4 Gene Polymorphism with Rheumatoid Arthritis [J]. J Guiyang Medical College, 2012, 37 (4): 372-375.
[51] Xu ZX, Chen JC, Qiu MS, et al. Association of five novel single nucleotide polymorphisms with rheumatoid arthritis suscepti-bility in the Han ethnicity in Xiamen area [J]. J Guangdong Med, 2016, 37 (7): 1003-1006.

[52] Park Y, Lee HS, Park Y, et al. Evidence for the role of STAT4 as ageneral autoimmunity locus in the Korean population [J]. Diabetes Metab Res Rev, 2011, 27 (8): 861-871.

[53] Zhao YJ, Liang WF, He MF, et al. Research on Correlation between the Graves Disease in the Han Chinese of Shanxi Area [J]. China \&Foreign Med Treat, 2016, 14 (1): 54-55, 58.

[54] Gao Xueren, Wang Jianguo, Yu Yongguo. The Association Between STAT4 rs7574865 Polymorphism and the Susceptibility of Autoimmune Thyroid Disease: A Meta-Analysis [J]. Frontiers in Genetics, 2018, 9: 708. 